\title{
Low-energy doubly virtual Compton scattering from dilepton electroproduction on a nucleon
}

\author{
Vladyslav Pauk, ${ }^{1}$ Carl E. Carlson, ${ }^{2}$ and Marc Vanderhaeghen ${ }^{1}$ \\ ${ }^{1}$ Institut für Kernphysik, Cluster of Excellence PRISMA ${ }^{+}$, Johannes Gutenberg-Universität, D-55099 Mainz, Germany \\ ${ }^{2}$ College of William and Mary, Physics Department, Williamsburg, Virginia 23187, USA
}

(Received 31 January 2020; revised 30 March 2020; accepted 22 July 2020; published 8 September 2020)

\begin{abstract}
We propose a new way to experimentally determine the subleading low-energy structure constant of doubly virtual Compton scattering on a proton. Such empirical determination will reduce the theoretical model error in estimates of the hadronic correction to the muonic hydrogen Lamb shift. We demonstrate that the dilepton forward-backward asymmetry in the $e^{-} p \rightarrow e^{-} p e^{-} e^{+}$process, which can be accessed at electron scattering facilities, yields a large sensitivity to this so far unknown low-energy constant.
\end{abstract}

DOI: 10.1103/PhysRevC.102.035201

\section{INTRODUCTION}

Extractions of the proton charge radius from muonic hydrogen $(\mu \mathrm{H})$ Lamb shift measurements over the past decade $[1,2]$ have reported a highly precise proton-radius value, with more than an order of magnitude improvement in the precision. These results disagreed by around 5.6 standard deviations, with the values obtained from measurements of energy level shifts in electronic hydrogen [3] or from electron-proton elastic scattering experiments [4]. This socalled proton radius puzzle has spurred a lot of activity, see Refs. $[5,6]$ for reviews. A new round of experiments using electronic hydrogen spectroscopy $[7,8]$, as well as a new electron scattering experiment [9] are favoring the lower value of the radius consistent with the $\mu \mathrm{H}$ spectroscopy results, although a recent electronic hydrogen experiment [10] has also reported a large value of the proton charge radius. To fully clarify this situation, further experiments with electron beams [11], muon beams [12,13], or by a direct comparison of cross sections for $\gamma p \rightarrow e^{+} e^{-} p$ versus $\gamma p \rightarrow \mu^{+} \mu^{-} p$ [14], are presently planned or underway. With the next generation of high-precision experiments both in scattering and spectroscopy, the focus is now shifting to improve the precision on this fundamental nucleon structure quantity, as spelled out recently in Ref. [15]. Indeed, to extract the proton charge radius from $\mu \mathrm{H}$ Lamb shift measurements, which is at present the most precise method, the proton form factors, structure functions, and polarizabilities are all required as input in a quantitative understanding of the hadronic correction [16-18]. At present the theoretical uncertainty due to this correction, which is evaluated in a dispersive framework, is of the same

Published by the American Physical Society under the terms of the Creative Commons Attribution 4.0 International license. Further distribution of this work must maintain attribution to the author(s) and the published article's title, journal citation, and DOI. Funded by $S C O A P^{3}$. size as the $2 \mathrm{P}-2 \mathrm{~S} \mu \mathrm{H}$ Lamb shift experimental uncertainty, and is the main limitation when converting a value of the Lamb shift to a value for the proton radius.

The main part of this hadronic uncertainty results from the subtraction function entering the forward doubly virtual Compton scattering process. It corresponds to the situation where the photons in the Compton process have zero energy and finite virtuality. At second order in the photon virtuality, this function is constrained by the magnetic polarizability, which is determined experimentally [19]. To fourth order in the photon virtuality, one low-energy constant in this subtraction function is at present empirically unconstrained [20], and one relies on chiral effective field theory calculations $[17,21]$ or phenomenological estimates. We demonstrate in this work that this low-energy constant can be accessed experimentally through the forward-backward asymmetry in the $e^{-} p \rightarrow$ $e^{-} p e^{-} e^{+}$process. A pioneering measurement of this forwardbackward asymmetry in the $\gamma p \rightarrow e^{-} e^{+} p$ process has been performed at DESY quite some time ago [22] as a test of the Kramers-Kronig relation at high energies. In the present work, we demonstrate that the corresponding experiments with a spacelike initial virtual photon, which can be realized at electron scattering facilities, yield a large sensitivity to this so far unknown low-energy constant.

\section{DOUBLY VIRTUAL COMPTON SCATTERING}

The helicity averaged forward doubly virtual Compton scattering process (VVCS), $\gamma^{*}(q)+N(p) \rightarrow \gamma^{*}(q)+N(p)$ is described by two invariant amplitudes, denoted by $T_{1}$ and $T_{2}$, which are functions of two kinematic invariants: $Q^{2}=$ $-q^{2}$ and $v=q \cdot p / M$, with $M$ the nucleon mass. Its covariant tensor structure in the four-vector indices of initial $(\mu)$ and final (v) photons can be written, following notations from Ref. [23], as:

$$
\alpha_{\mathrm{em}} M^{\mu \nu}(\mathrm{VVCS}) \equiv \hat{g}^{\mu \nu} T_{1}\left(\nu, Q^{2}\right)-\frac{\hat{p}^{\mu} \hat{p}^{\nu}}{M^{2}} T_{2}\left(\nu, Q^{2}\right),
$$


TABLE I. Values of the $Q^{4}$ term of the subtraction function $\bar{T}_{1}\left(0, Q^{2}\right)$ (second column) and of the dVCS low-energy constant $b_{3,0}$ (third column), both in units $10^{-4} \mathrm{fm}^{5}$, in different theoretical approaches [20]. The indicated range for the HBChPT result corresponds with the range given by Eq. (15) in Ref. [17].

\begin{tabular}{lccc}
\hline \hline Source & Ref. & $\frac{1}{2} \bar{T}_{1}^{\prime \prime}(0)$ & $\alpha_{\mathrm{em}} b_{3,0}$ \\
\hline HBChPT & {$[17]$} & {$[-1.01,-0.35]$} & \\
$\pi N$ loops & & -0.06 & 0.001 \\
$\pi \Delta$ loops & & -0.10 & -0.005 \\
$\Delta$ exchange & & -1.98 & 0.11 \\
Total BChPT & {$[20]$} & $-2.14 \pm 0.98$ & $0.11 \pm 0.05$ \\
superconvergence SR & {$[24]$} & -0.47 & 3.96 \\
\hline \hline
\end{tabular}

with $\quad \hat{g}^{\mu \nu} \equiv g^{\mu \nu}-q^{\mu} q^{v} / q^{2}, \quad \hat{p}^{\mu} \equiv p^{\mu}-p \cdot q / q^{2} q^{\mu}, \quad$ and where $\alpha_{\mathrm{em}}=e^{2} / 4 \pi \approx 1 / 137$. The optical theorem relates the imaginary parts of $T_{1}$ and $T_{2}$ as:

$$
\operatorname{Im} T_{1}\left(v, Q^{2}\right)=\frac{e^{2}}{4 M} F_{1}, \quad \operatorname{Im} T_{2}\left(v, Q^{2}\right)=\frac{e^{2}}{4 v} F_{2},
$$

where $F_{1}, F_{2}$ are the conventionally defined structure functions parametrizing inclusive electron-nucleon scattering, and depend on $Q^{2}$ and $x \equiv Q^{2} / 2 M \nu$. The two-photon exchange correction to the $\mu \mathrm{H}$ Lamb shift can be expressed as a weighted double integral over $Q^{2}$ and $v$ of the forward amplitudes $T_{1}$ and $T_{2}$ [16]. Using the empirical input of $F_{1}$ and $F_{2}$, the $v$ dependence of $T_{2}$ has been fully reconstructed in Ref. [16] using an unsubtracted dispersion relation, whereas the dispersion relation for $T_{1}$ requires one subtraction, which can be chosen at $v=0$ as $T_{1}\left(0, Q^{2}\right)$. The subtraction function is usually split in a Born part (corresponding with the nucleon intermediate state), and a remainder, so-called non-Born part, which we denote in the following by $\bar{T}_{1}\left(0, Q^{2}\right)$. Although the Born part can be expressed in terms of elastic form factors (see Ref. [23] for the corresponding expression), the non-Born part cannot be fixed empirically so far. In general, one can, however, write down a low $Q^{2}$ expansion of $\bar{T}_{1}\left(0, Q^{2}\right)$ as:

$$
\bar{T}_{1}\left(0, Q^{2}\right)=\beta_{M 1} Q^{2}+\frac{1}{2} T_{1}^{\prime \prime}(0) Q^{4}+O\left(Q^{6}\right),
$$

where the term proportional to $Q^{2}$ is empirically determined by the magnetic dipole polarizability $\beta_{M 1}$ [19]. Theoretical estimates for the subtraction term were given at order $Q^{4}$ in heavy-baryon chiral perturbation theory (HBChPT) [17], and covariant baryon chiral perturbation theory (BChPT), both at leading order (LO) due to $\pi N$ loops, and at next-to-leading order (NLO), including both $\Delta$ (1232)-exchange and $\pi \Delta$ loops $[20,21]$. Furthermore, the subtraction function was also extracted from superconvergence sum rule (SR) relations [24]. The different estimates for $\bar{T}_{1}^{\prime \prime}(0)$ are compared in Table I. Even for these theoretically well-motivated approaches, the spread among the different estimates is quite large. The resulting uncertainty due to this subtraction term constitutes at present the main uncertainty in the theoretical Lamb shift estimate. We next discuss how to avoid such model dependence, and propose an empirical way to determine $\bar{T}_{1}^{\prime \prime}(0)$.

\section{OBSERVABLES}

To empirically access the $Q^{4}$ term and potentially also higher-order terms in $\bar{T}_{1}\left(0, Q^{2}\right)$, we consider the full offforward doubly virtual Compton process, $\gamma^{*}(q)+N(p) \rightarrow$ $\gamma^{*}\left(q^{\prime}\right)+N\left(p^{\prime}\right)$, where both photons are virtual. In the following, we study the case where the initial photon is spacelike $\left(q^{2}<0\right)$, and the final photon is timelike $\left(q^{\prime 2}>0\right)$, which can be accessed experimentally. In general, the full off-forward doubly virtual Compton scattering (dVCS) amplitude off a proton is described by 18 tensor structures in the initial $(\mu)$ and final $(v)$ photon four-vector indices [25]. In this work, we will only need the helicity-averaged amplitude, which is described by five independent tensors, and can be expressed as [26]:

$$
M^{\mu \nu}=\sum_{i=1,2,3,4,19} B_{i}\left(\nu, q^{2}, q^{\prime 2}, q \cdot q^{\prime}\right) T_{i}^{\mu \nu},
$$

where $T_{i}^{\mu \nu}$ are the spin-independent and gauge invariant tensors, symmetric under exchange of the two virtual photons, see Eq. (8) in Ref. [20] for the corresponding expressions. Furthermore, in (4), the invariant amplitudes $B_{i}$ are functions of four Lorentz invariants, with $v \equiv q \cdot P / M$, where $P \equiv(p+$ $\left.p^{\prime}\right) / 2$. As the forward VVCS process of Eq. (1) is a special case of Eq. (4), one can express the subtraction function as [20]:

$$
\bar{T}_{1}\left(0, Q^{2}\right)=\alpha_{\mathrm{em}} Q^{2}\left(\bar{B}_{1}+Q^{2} \bar{B}_{3}\right),
$$

where both non-Born amplitudes $\bar{B}_{1}, \bar{B}_{3}$ are understood in the forward limit $\left(q=q^{\prime}\right)$, i.e., $\bar{B}_{i}\left(0, q^{2}, q^{2}, q^{2}\right)$ for $i=1,3$.

The Born contribution was worked out in Ref. [25]. For the helicity averaged amplitude, it only contributes to the amplitudes $B_{1}$ and $B_{2}$, see Eq. (8) in Ref. [20]. The non-Born part of the dVCS amplitudes, denoted as $\bar{B}_{i}$, can be expanded for small values of $q^{2}, q^{\prime 2}, q \cdot q^{\prime}$ and $\nu$, with coefficients given by polarizabilities. As the $v$ dependence can be fully reconstructed up to at most one subtraction, through a dispersion relation, we only need to discuss the amplitudes entering the subtraction function. To determine $\bar{T}_{1}\left(0, Q^{2}\right)$ up to the $Q^{4}$ term, we use the low-energy expansion in $k \in\left\{q, q^{\prime}\right\}$ [20]:

$$
\begin{aligned}
& \bar{B}_{1}\left(0, q^{2}, q^{\prime 2}, q \cdot q^{\prime}\right) \\
& =\frac{1}{\alpha_{\mathrm{em}}}\left\{\beta_{M 1}-\frac{1}{6} \beta_{M 2} q \cdot q^{\prime}\right. \\
& \left.\quad-\left(\beta_{M 1}^{\prime}(0)+\frac{\beta_{M 1}}{8 M^{2}}\right)\left(q^{2}+q^{\prime 2}\right)\right\}+O\left(k^{4}\right), \\
& \bar{B}_{3}\left(0, q^{2}, q^{\prime 2}, q \cdot q^{\prime}\right)=b_{3,0}+O\left(k^{2}\right),
\end{aligned}
$$

where $\beta_{M 2}$ is the magnetic quadrupole polarizability determined from real Compton scattering [27], and $\beta_{M 1}^{\prime}(0)$ is the slope at $Q^{2}=0$ of the generalized magnetic dipole polarizability, which is accessed through virtual Compton scattering, see Ref. [28] for a recent review. The low-energy constant $b_{3,0}$ is not determined empirically so far because the tensor structure $T_{3}^{\mu \nu}$ decouples when either the initial or final photon is real. Using Eq. (6), we can express the $Q^{4}$ term in Eq. (3) as:

$$
\frac{1}{2} T_{1}^{\prime \prime}(0)=\frac{1}{6} \beta_{M 2}+2 \beta_{M 1}^{\prime}(0)+\frac{\beta_{M 1}}{4 M^{2}}+\alpha_{\mathrm{em}} b_{3,0} .
$$




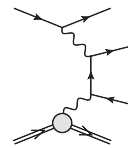

(a)

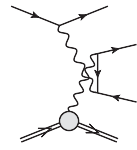

(b)

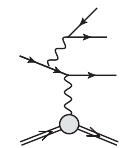

(c)

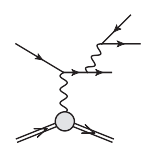

(d)

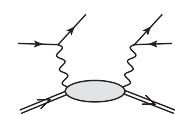

(e)
FIG. 1. Feynman diagrams for the spacelike BH process [(a) and (b)], the timelike BH process [(c) and (d)], and the dVCS process [diagram (e)]. The single (double) lines correspond with leptons (nucleons).

We compare in Table I (third column) several theoretically motivated estimates for $b_{3,0}$ : the BChPT including $\Delta$ pole corresponds with a very small value of $b_{3,0}$ in comparison with a superconvergence SR estimate for $T_{1}^{\prime \prime}(0)$.

To empirically determine $b_{3,0}$ we consider the process of electroproduction of a dilepton pair on the nucleon,

$$
e^{-}(k)+N(p) \rightarrow e^{-}\left(k^{\prime}\right)+N\left(p^{\prime}\right)+e^{-}\left(l_{-}\right)+e^{+}\left(l_{+}\right),
$$

where the four-momenta of the corresponding particles are shown in parentheses. We define the eightfold phase space of the reaction (8) in terms of five invariants:

$$
\begin{aligned}
& s=(k+p)^{2}, \quad Q^{2}=-\left(k-k^{\prime}\right)^{2}, \quad W^{2}=(q+p)^{2}, \\
& t=\left(p^{\prime}-p\right)^{2}, \quad q^{\prime 2}=\left(l_{-}+l_{+}\right)^{2},
\end{aligned}
$$

and three angles $\Phi, \theta_{l}$, and $\phi_{l}$. The invariant $s$ is obtained from the electron beam energy $E_{e}$ as $s=M^{2}+2 M E_{e}, \Phi$ is the angle of the initial electron plane relative to the production plane, spanned by the vectors $q \equiv k-k^{\prime}$ and $q^{\prime} \equiv l_{-}+l_{+}$ in the c.m. frame $(\vec{q}+\vec{p}=0)$, and $\theta_{l}, \phi_{l}$ are the angles of the produced negative lepton in the dilepton rest frame (with polar angle defined relative to the c.m. direction of $q^{\prime}$ ). The differential cross section of the reaction (8) reads

$$
\begin{aligned}
\frac{d \sigma}{d Q^{2} d W^{2} d \Phi d t d q^{\prime 2}\left(d \Omega_{l}\right)_{e^{-} e^{+}}} \\
=\frac{1}{(4 \pi)^{7}} \frac{1}{2\left(s-M^{2}\right)^{2}} \\
\quad \times \frac{\left(1-4 m_{e}^{2} / q^{\prime 2}\right)^{1 / 2}}{\lambda\left(W^{2}, M^{2},-Q^{2}\right)^{1 / 2}} \overline{\sum_{i}} \sum_{f}|\mathcal{M}|^{2},
\end{aligned}
$$

where $m_{e}$ is the lepton mass, $\lambda$ is the Källén triangle function, and $\mathcal{M}$ stands for the amplitude of the reaction (8).

At $O\left(\alpha_{O E D}^{2}\right)$, the reaction (8) is described by the processes shown in Fig. 1. The first four diagrams correspond with the spacelike (SL) and timelike (TL) Bethe-Heitler (BH) processes, whereas the last is the dVCS process. The BH-SL and BH-TL diagrams are fully determined by the nucleon's electromagnetic FFs. We adopt the FF parametrization of [4] in the following, which we can analytically continue to the small timelike virtualities considered here. The invariant amplitude for the dVCS process $\left(\mathcal{M}_{C}\right)$, where initial (final) photons have spacelike (timelike) virtualities, is given by

$$
\mathcal{M}_{C}=\frac{i e^{4}}{q^{\prime 2} Q^{2}} \bar{N}\left(p^{\prime}\right) M^{\mu \nu} N(p) \bar{u}\left(k^{\prime}\right) \gamma_{\mu} u(k) \bar{u}\left(l_{-}\right) \gamma_{\nu} v\left(l_{+}\right),
$$

where $M^{\mu \nu}$ is the dVCS tensor of Eq. (4). To access the real part of the dVCS amplitude and empirically extract the
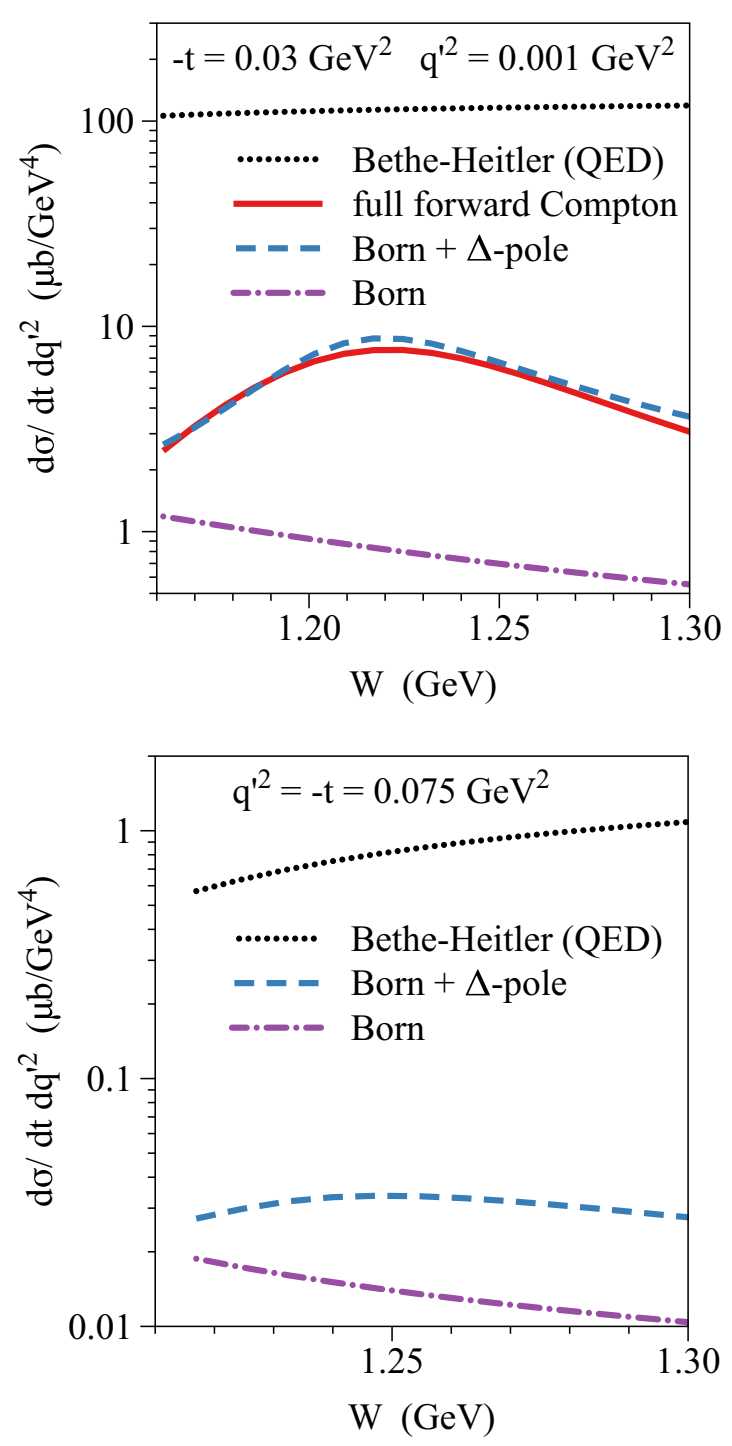

FIG. 2. $W$ dependence of the $\gamma p \rightarrow e^{-} e^{+} p$ cross section integrated over the dilepton angles for two kinematic settings. The $\mathrm{BH}$ cross section is compared with models for the Compton cross section, and a full calculation based on the empirical forward structure functions [30] for the near-forward quasireal Compton kinematics.

low-energy constant $b_{3,0}$ we consider the forward-backward asymmetry $A_{F B}$, defined in the dilepton rest frame as:

$$
A_{F B} \equiv \frac{\overline{\sum_{i}} \sum_{f}\left\{|\mathcal{M}|_{\theta_{l}, \phi_{l}}^{2}-|\mathcal{M}|_{\pi-\theta_{l}, \phi_{l}+\pi}^{2}\right\}}{\overline{\sum_{i} \sum_{f}\left\{|\mathcal{M}|_{\theta_{l}, \phi_{l}}^{2}+|\mathcal{M}|_{\pi-\theta_{l}, \phi_{l}+\pi}^{2}\right\}}} .
$$

The only nonzero contribution to this observable comes from the interference between processes with an even (BHSL) and odd (BH-TL and dVCS) number of photon couplings to the dilepton pair, due to charge conjugation. Explicitly, the numerator in Eq. (11) is proportional to $\Re\left[\mathcal{M}_{B H-S L}\left(\mathcal{M}_{B H-T L}+\mathcal{M}_{C}\right)^{*}\right]$. 


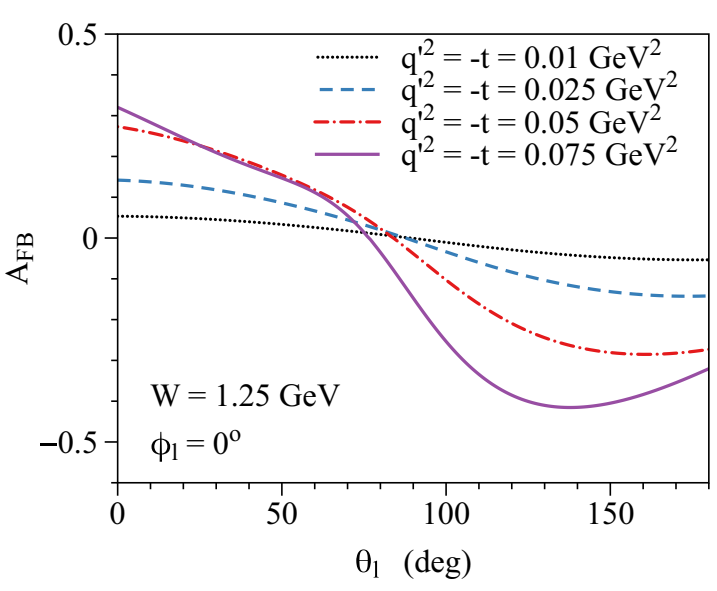

FIG. 3. Lepton angular dependence (in dilepton c.m. frame, for $\phi_{l}=0^{\circ}$ ) of the $\gamma p \rightarrow e^{-} e^{+} p$ asymmetry $A_{F B}$ for $W=1.25 \mathrm{GeV}$, and for different values of $q^{\prime 2}$ and $-t$.

\section{RESULTS AND DISCUSSION}

We start our discussion by considering the case of an initial real photon through the $\gamma p \rightarrow e^{-} e^{+} p$ reaction. In Fig. 2 we show the dependence of the $\gamma p \rightarrow e^{-} e^{+} p$ cross section on the c.m. energy $W$ for two settings. One of kinematics is approaching the forward Compton process (top panel), with $q^{\prime 2}$ and $t$ values near the ones considered in the experiment of Ref. [22]. To provide a model of the inelastic effects in the dVCS process we consider an effective description of the non-Born part of the dVCS amplitude in the $\Delta$ (1232) region by the $\Delta$-pole amplitude. For the electromagnetic $N \rightarrow \Delta$ transition we use the empirical parametrization, see Ref. [29]. To estimate the accuracy of the description, we also implemented for the near-forward real Compton situation, with $-t$ small and $q^{\prime 2}$ very close to zero, a full dispersive calculation based on empirical structure functions [30], consistent with the so far only data point for $A_{F B}$ [22]. We see from Fig. 2 that around c.m. energy $W=1.25 \mathrm{GeV}$ the Compton cross section integrated over the dilepton solid angle is reproduced by the Born $+\Delta$-pole description within an accuracy of $5 \%$ or better. Furthermore, for larger values of $q^{\prime 2}=-t$, the ratio between $\mathrm{BH}$ and Compton cross sections decreases. Therefore, one expects an increase of $A_{F B}$ with increasing values of $q^{\prime 2}$ and $-t$, as is demonstrated in the lepton angular dependence in Fig. 3. One sees that for $q^{\prime 2}=-t=0.075 \mathrm{GeV}^{2}, A_{F B}$ reaches values between $-40 \%$ and $+30 \%$.

Having assessed the sensitivity of $A_{F B}$ to the dVCS amplitude for real photons, we next extend it to the initial virtual photon case through the $e^{-} p \rightarrow e^{-} p e^{-} e^{+}$reaction. As $A_{F B}$ depends on the real part of the dVCS amplitude, it holds promise to study the sensitivity on the low-energy constant $b_{3,0}$. In Fig. 4 , we show the cross section and $A_{F B}$ for the $e^{-} p \rightarrow e^{-} p e^{-} e^{+}$process at $W=1.25 \mathrm{GeV}$, where the $\Delta$ pole was found to yield a very good description of the total Compton result, and in kinematics where $Q^{2}=q^{\prime 2}=-t=$ $0.075 \mathrm{GeV}^{2}$.

One notices that in the forward angular range the BetheHeitler process yields only a small asymmetry. In these
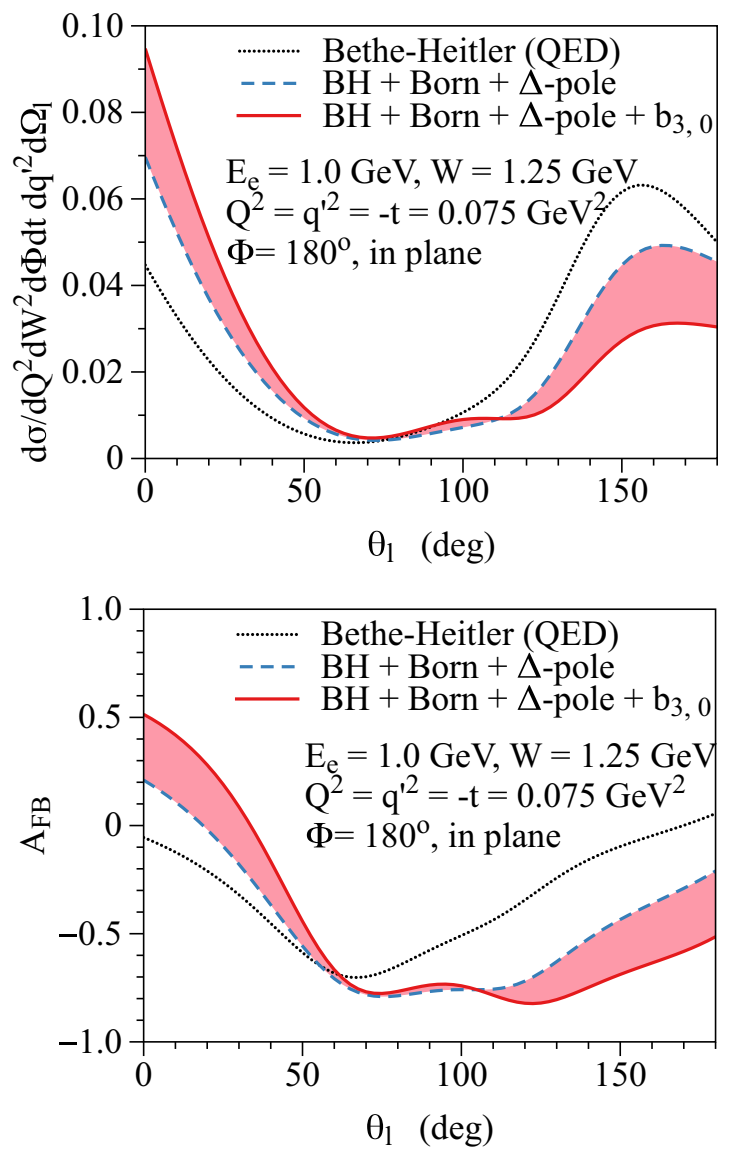

FIG. 4. Lepton angular dependence (in dilepton c.m. frame) of the $e^{-} p \rightarrow e^{-} p e^{-} e^{+}$differential cross section (top panel, in units $\mathrm{nb} / \mathrm{GeV}^{8} \mathrm{sr}^{2}$ ) and asymmetry $A_{F B}$ (bottom panel). The red band shows the sensitivity on the low-energy constant $b_{3,0}$, corresponding with the spread in Table I.

ranges, the Compton process yields a large change in the asymmetry, up to $50 \%$. The red band shows the sensitivity on $b_{3,0}$, corresponding with the spread in Table I. For forward angles, where the sensitivity is the largest, the spread in the values for the subtraction constant $T_{1}^{\prime \prime}(0)$ in Table I, corresponds with a change in $A_{F B}$ from $20 \%-50 \%$.

We can obtain an initial estimate as to the experimental feasibility from dilepton electroproduction experiments already performed at MAMI [31-33] and JLab [34]. A pilot MAMI experiment, with the aim of searching for dark photons through dilepton electroproduction on a nuclear target, has been performed for a dilepton invariant mass of $250 \mathrm{MeV}$, i.e., $q^{\prime 2} \approx 0.06 \mathrm{GeV}^{2}$, in the range considered in the present work. The followup dark photon experiment of Ref. [32] had measured 22 such settings. The pilot experiment has achieved a luminosity of $L=8 \times 10^{38} \mathrm{~cm}^{-2} \mathrm{~s}^{-1}$ on a nuclear target $(Z=73)$ [31], which corresponds for a proton target and one day of beam time to an integrated luminosity of $L_{\text {int, } 1 \text { day }} \approx 10$ $\mathrm{fb}^{-1}$. We can thus estimate the count rate on the nucleon for a 100 -day experiment to be of the order $N \approx 100$, which yields a $10 \%$ statistical accuracy on $A_{F B}$ in Fig. 4. As the difference between both estimates for $b_{3,0}(0.11$ vs. 3.96 in the units of 

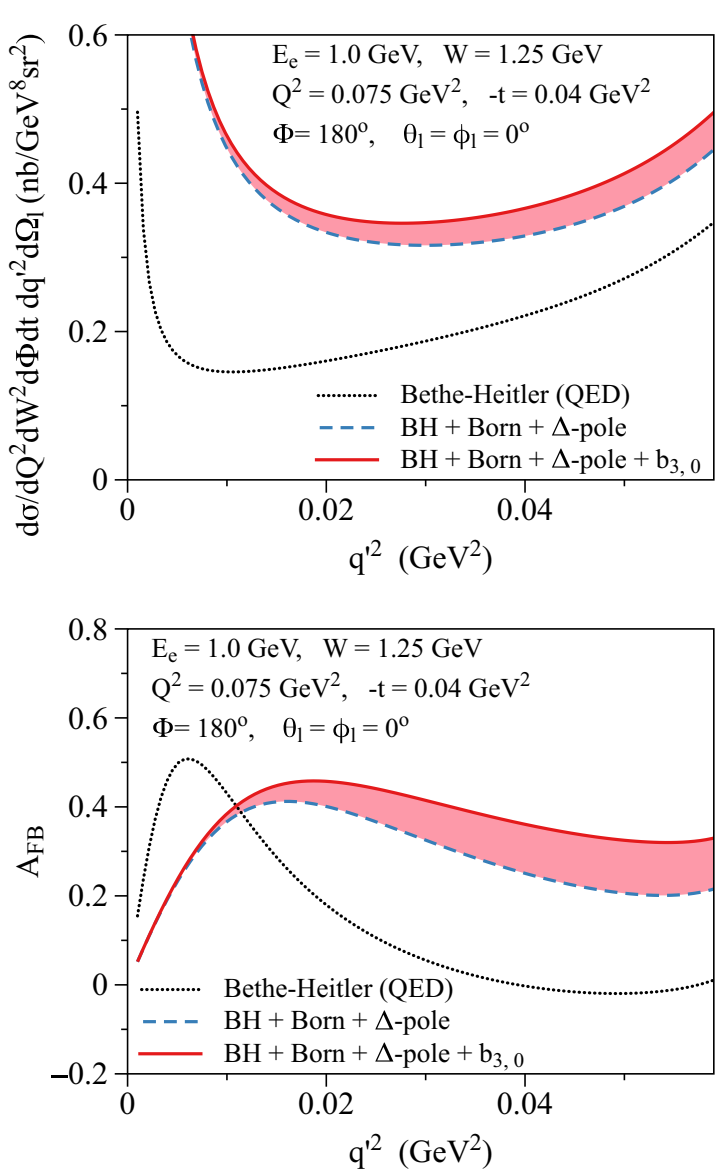

FIG. 5. Dependence on the squared dilepton invariant mass $q^{\prime 2}$ of the $e^{-} p \rightarrow e^{-} p e^{-} e^{+}$differential cross section (top panel, in units $\mathrm{nb} / \mathrm{GeV}^{8} \mathrm{sr}^{2}$ ) and asymmetry $A_{F B}$ (bottom panel). The red band shows the sensitivity on the low-energy constant $b_{3,0}$, corresponding with the spread in Table I.

Table I), yields a change in the asymmetry $A_{F B}$ of around $30 \%$, a measurement of $A_{F B}$ with a precision of around $10 \%$ would yield an extraction of $b_{3,0}$ with a $33 \%$ precision. For the planning of such experiment, a further optimization is surely called for to increase the count rates, and the present calculation will serve for such optimization study. As a first step towards this goal, we show in Fig. 5 our estimate for the same value of $Q^{2}$ but about half the value for $-t$ as function of the squared dilepton virtuality $q^{\prime 2}$. We see that around $q^{\prime 2}=0.05 \mathrm{GeV}^{2}$ the cross section is around four times higher compared to the kinematical situation considered in Fig. 4, which would yield a statistical precision on $A_{F B}$ of around $5 \%$ for a 100-day experiment. For such kinematics the bottom panel shows that the difference between both estimates for $b_{3,0}$ yields a change in the asymmetry $A_{F B}$ of around $15 \%$, which would also result in an extraction of $b_{3,0}$ with a $33 \%$ precision.

\section{SUMMARY}

In summary, we have explored a direct empirical determination of the subtraction function in the doubly virtual Compton process on a nucleon, which corresponds at present with the leading uncertainty in the hadronic correction to muonic atom spectroscopy. To fourth order in the photon virtuality, one low-energy quantity in the subtraction function is so far empirically unconstrained. We have demonstrated that it can be accessed experimentally through the forward-backward asymmetry of the $e^{-} p \rightarrow e^{-} p e^{-} e^{+}$process. This observable is directly sensitive to the interference between the QED and dVCS amplitudes. Different theoretical estimates for this low-energy constant induce a change between $20 \%$ and $50 \%$ in the corresponding asymmetry in the $\Delta(1232)$ region. This observable can be accessed by precision experiments at the electron facilities MAMI, MESA, and JLab.

\section{ACKNOWLEDGMENTS}

This work was supported by the Deutsche Forschungsgemeinschaft (DFG, German Research Foundation), through the Collaborative Research Center [The Low-Energy Frontier of the Standard Model, Projektnummer 204404729 SFB 1044], and through the Cluster of Excellence (Precision Physics, Fundamental Interactions, and Structure of Matter) (PRISMA+ EXC 2118/1) within the German Excellence Strategy (Project ID 39083149). C.E.C. thanks the National Science Foundation (USA) for support under grant PHY1812326, and the Johannes Gutenberg-University, and the Nordic Institute for Theoretical Physics (NORDITA) for hospitality while this work was underway.
[1] R. Pohl, A. Antognini, F. Nez, F. D. Amaro, F. Biraben, J. M. R. Cardoso, D. S. Covita, A. Dax et al., Nature (London) 466, 213 (2010).

[2] A. Antognini, F. Nez, K. Schuhmann, F. D. Amaro, F. Biraben, J. M. R. Cardoso, D. S. Covita, A. Dax et al., Science 339, 417 (2013).

[3] P. J. Mohr, D. B. Newell, and B. N. Taylor, Rev. Mod. Phys. 88, 035009 (2016).

[4] J. C. Bernauer et al. (A1 Collaboration), Phys. Rev. Lett. 105, 242001 (2010); Phys. Rev. C 90, 015206 (2014).

[5] R. Pohl, R. Gilman, G. A. Miller, and K. Pachucki, Ann. Rev. Nucl. Part. Sci. 63, 175 (2013).

[6] C. E. Carlson, Prog. Part. Nucl. Phys. 82, 59 (2015).
[7] A. Beyer et al., Science 358, 79 (2017).

[8] N. Bezginov, T. Valdez, M. Horbatsch, A. Marsman, A. C. Vutha, and E. A. Hessels, Science 365, 1007 (2019).

[9] W. Xiong et al., Nature (London) 575, 147 (2019).

[10] H. Fleurbaey, S. Galtier, S. Thomas, M. Bonnaud, L. Julien, F. Biraben, F. Nez, M. Abgrall, and J. Guena, Phys. Rev. Lett. 120, 183001 (2018).

[11] A. Denig, J. Univ. Sci. Tech. China 46, 608 (2016).

[12] MUSE Collaboration, R. Gilman, arXiv:1709.09753 [physics.ins-det].

[13] B. Adams et al., arXiv:1808.00848 [hep-ex].

[14] V. Pauk and M. Vanderhaeghen, Phys. Rev. Lett. 115, 221804 (2015). 
[15] H. W. Hammer and U. G. Meissner, Science Bulletin 65, 257 (2020).

[16] C. E. Carlson and M. Vanderhaeghen, Phys. Rev. A 84, 020102(R) (2011).

[17] M. C. Birse and J. A. McGovern, Eur. Phys. J. A 48, 120 (2012).

[18] A. Antognini, F. Kottmann, F. Biraben, P. Indelicato, F. Nez, and R. Pohl, Ann. Phys. (NY) 331, 127 (2013).

[19] M. Tanabashi et al. (Particle Data Group), Phys. Rev. D 98, 030001 (2018).

[20] V. Lensky, F. Hagelstein, V. Pascalutsa, and M. Vanderhaeghen, Phys. Rev. D 97, 074012 (2018).

[21] J. M. Alarcon, V. Lensky, and V. Pascalutsa, Eur. Phys. J. C 74, 2852 (2014).

[22] H. Alvensleben et al., Phys. Rev. Lett. 30, 328 (1973).

[23] B. Pasquini and M. Vanderhaeghen, Ann. Rev. Nucl. Part. Sci. 68, 75 (2018).

[24] O. Tomalak and M. Vanderhaeghen, Eur. Phys. J. C 76, 125 (2016).
[25] R. Tarrach, Nuovo Cim. A 28, 409 (1975).

[26] D. Drechsel, G. Knochlein, A. Y. Korchin, A. Metz, and S. Scherer, Phys. Rev. C 57, 941 (1998).

[27] B. R. Holstein, D. Drechsel, B. Pasquini, and M. Vanderhaeghen, Phys. Rev. C 61, 034316 (2000).

[28] H. Fonvieille, B. Pasquini, and N. Sparveris, Progress Particle Nuclear Physics 113, 103754 (2020).

[29] V. Pascalutsa, M. Vanderhaeghen, and S. N. Yang, Phys. Rept. 437, 125 (2007).

[30] O. Gryniuk, F. Hagelstein, and V. Pascalutsa, Phys. Rev. D 92 , 074031 (2015).

[31] H. Merkel et al. (A1 Collaboration), Phys. Rev. Lett. 106, 251802 (2011).

[32] H. Merkel et al. (A1 Collaboration), Phys. Rev. Lett. 112, 221802 (2014).

[33] T. Beranek, H. Merkel, and M. Vanderhaeghen, Phys. Rev. D 88, 015032 (2013).

[34] S. Abrahamyan et al. (APEX Collaboration), Phys. Rev. Lett. 107, 191804 (2011). 